\title{
Stress state of hinged supported thin-wall elastic structures
}

\author{
Lilya Kharasova*1[0000-0002-4713-3930], and Samat Timergaliev2[0000-0003-4345-622X] \\ ${ }^{1}$ Kazan Federal University, Naberezhnye Chelny Institute, 420008 Naberezhnye Chelny, Russia \\ ${ }^{2}$ Kazan State University of Architecture and Engineering, 420043 Kazan, Russia
}

\begin{abstract}
The paper studies the stress-strain state of flat elastic isotropic thin-walled shell structures in the framework of the S. P. Timoshenko shear model with pivotally supported edges. The stress-strain state of shell structures is described by a system of five second-order nonlinear partial differential equations under given static boundary conditions with respect to generalized displacements. The system of equations under study is linear in terms of tangential displacements, rotation angles, and nonlinear in terms of normal displacement. To find a solution to the system that satisfies the given static boundary conditions, integral representations for generalized displacements containing arbitrary holomorphic functions are used. Finding holomorphic functions is one of the main and difficult points in the proposed study. The integral representations constructed in this way allow us to reduce the original problem to a single nonlinear operator equation with respect to the deflection, the solvability of which is established using the principle of compressed maps.
\end{abstract}

Keywords. Building constructions of the shell type, stress-strain state, equilibrium equations, static boundary conditions, generalized displacements.

\section{Introduction}

Currently, there are a large number of works devoted to the strength of thin-walled shell structures, taking into account the geometric and/or physical nonlinearity [1-9]. This is due to the widespread use of elastic thin-walled shell structures in aviation, space technology, shipbuilding, mechanical engineering and construction. In very rare cases, nonlinear problems are solved in a closed form. For this reason, a wide range of approximate methods with the use of computers is used to solve them. When solving problems numerically, the problem of convergence of the numerical solution to the exact (real) solution of the problem always comes to the fore. The solution to this problem, as is known, is based on a rigorous mathematical study of the stress-strain state of thin-walled shell structures. At present, this problem is sufficiently fully studied in the framework of the simplest Kirchhoff-Love model [10-17]. Questions related to the qualitative study of the stress-strain state within the framework of more general models of the theory of thin-walled shell structures that do not rely on the Kirchhoff-Love hypotheses were included in the well-known list of unsolved problems of the mathematical theory of shells by I.I. Vorovich [10] and remained open until

*Corresponding author: kharasova.liya@mail.ru 
recently. To date, there are a number of works [18-24] devoted to the study of the stress-strain state in the framework of the Timoshenko shear model. The research in [18-24] is based on integral representations for generalized displacements containing arbitrary holomorphic functions that are found in such a way that the generalized displacements satisfy the given boundary conditions. Two approaches are used to build them. The first approach is based on the application of explicit representations of solutions to Riemann - Hilbert problems for holomorphic functions in the unit circle. Therefore, a flat region homeomorphic to the median surface of the shell is either assumed to be a unit circle from the very beginning [18-20], or conformally mapped to a unit circle [22], [24]. In the second approach, the theory of onedimensional singular integral equations is used to determine holomorphic functions [21], [23]. In this paper, the conformal mapping method is used to study a nonlinear problem for arbitrary flat shells under different boundary conditions.

\section{Materials and methods}

A system of five nonlinear partial differential equations of the second order of the following form is considered in an arbitrary bounded domain $\Omega$ :

$$
\begin{gathered}
w_{1 \alpha^{1} \alpha^{1}}+\mu_{1} w_{1 \alpha^{2} \alpha^{2}}+\mu_{2} w_{2 \alpha^{1} \alpha^{2}}=f_{1}, \\
\mu_{1} w_{2 \alpha^{1} \alpha^{1}}+w_{2 \alpha^{2} \alpha^{2}}+\mu_{2} w_{1 \alpha^{1} \alpha^{2}}=f_{2}, \\
k^{2} \mu_{1}\left(w_{3 \alpha^{1} \alpha^{1}}+w_{3 \alpha^{2} \alpha^{2}}+\psi_{1 \alpha^{1}}+\psi_{2 \alpha^{2}}\right)+k_{3} w_{1 \alpha^{1}}+k_{4} w_{2 \alpha^{2}}-k_{5} w_{3}+ \\
+k_{3} w_{3 \alpha^{1}}^{2} / 2+k_{4} w_{3 \alpha^{2}}^{2} / 2+\beta_{2}\left[\left(T^{\lambda \mu} w_{3 \alpha^{2}}\right)_{\alpha^{\mu}}+R^{3}\right]=0, \\
\psi_{1 \alpha^{1} \alpha^{1}}+\mu_{1} \psi_{1 \alpha^{2} \alpha^{2}}+\mu_{2} \psi_{2 \alpha^{1} \alpha^{2}}=g_{1}+k_{0} \psi_{1}, \\
\mu_{1} \psi_{2 \alpha^{1} \alpha^{1}}+\psi_{2 \alpha^{2} \alpha^{2}}+\mu_{2} \psi_{1 \alpha^{1} \alpha^{2}}=g_{2}+k_{0} \psi_{2}
\end{gathered}
$$

under the following conditions:

$$
\begin{gathered}
w_{2}=w_{3}=\Psi_{2}=0 \\
\left(w_{1 \alpha^{1}}+\mu w_{2 \alpha^{2}}\right)(t) d \alpha^{2} / d s-\mu_{1}\left(w_{1 \alpha^{2}}+w_{2 \alpha^{1}}\right)(t) d \alpha^{1} / d s=\varphi_{1}\left(w_{3}\right)(t), \\
\left(\psi_{1 \alpha^{1}}+\mu \psi_{2 \alpha^{2}}\right)(t) d \alpha^{2} / d s-\mu_{1}\left(\psi_{1 \alpha^{2}}+\psi_{2 \alpha^{1}}\right)(t) d \alpha^{1} / d s=\widetilde{\varphi}_{1}\left(w_{3}\right)(t),
\end{gathered}
$$

on its border $\Gamma$.

The following designations are adopted in the formulas (1)-(4):

$$
\begin{gathered}
f_{1} \equiv f_{1}\left(w_{3}\right)=k_{3} w_{3 \alpha^{1}}-w_{3 \alpha^{1}} w_{3 \alpha^{1} \alpha^{1}}-\mu_{2} w_{3 \alpha^{2}} w_{3 \alpha^{1} \alpha^{2}}-\mu_{1} w_{3 \alpha^{1}} w_{3 \alpha^{2} \alpha^{2}}-\beta_{2} R^{1}, \\
f_{2} \equiv f_{2}\left(w_{3}\right)=k_{4} w_{3 \alpha^{2}}-w_{3 \alpha^{2}} w_{3 \alpha^{2} \alpha^{2}}-\mu_{2} w_{3 \alpha^{1}} w_{3 \alpha^{1} \alpha^{2}}-\mu_{1} w_{3 \alpha^{2}} w_{3 \alpha^{1} \alpha^{1}}-\beta_{2} R^{2}, \\
g_{j} \equiv g_{j}\left(w_{3}\right)=k_{0} w_{3 \alpha^{j}}-\beta_{1} L^{j}, j=1,2, \mu_{1}=(1-\mu) / 2, \mu_{2}=(1+\mu) / 2, \\
\varphi_{1}\left(w_{3}\right)(t)=\beta_{2} P^{1}(s)-\left[w_{3 \alpha^{1}}^{2}(t) / 2+\mu w_{3 \alpha^{2}}^{2}(t) / 2\right] d \alpha^{2} / d s+\mu_{1} w_{3 \alpha^{1}}(t) w_{3 \alpha^{2}}(t) d \alpha^{1} / d s, \\
\widetilde{\varphi}_{1}\left(w_{3}\right)(t)=\beta_{1} N^{1}(s), t=t(s)=\alpha^{1}(s)+i \alpha^{2}(s), k_{3}=k_{1}+\mu k_{2}, k_{4}=k_{2}+\mu k_{1}, \\
k_{5}=k_{1}^{2}+k_{2}^{2}+2 \mu k_{1} k_{2}, k_{0}=6 k^{2}(1-\mu) / h^{2}, \beta_{1}=12\left(1-\mu^{2}\right) /\left(h^{3} E\right), \beta_{2}=\left(1-\mu^{2}\right) /(E h) .
\end{gathered}
$$

The system (1), together with boundary conditions (2)-(4), describes the equilibrium state of an elastic shallow isotropic homogeneous shell with hinged edges within the shear model by S.P. Timoshenko [25, p. 168-170,269]. In this case: $T^{\lambda \mu}$ - the efforts $(\lambda, \mu=1,3) ; w_{j}(j=1,2)$ and $w_{3}$ - tangential and normal displacement of the points $S_{0}, \Psi_{j}(j=1,2)$ the angles of normal section rotation $S_{0}, R^{j}\left(j=\overline{1,3)}, L^{k}(k=1,2), N^{l}, P^{l}\right.$ the components of external forces acting on the shell, $\mu=$ const - Poisson's ratio, $E=$ const - Young's modulus, $k_{1}, k_{2}=$ const - principal curvatures, $k_{2}=$ const - shear coefficient, $h=$ const - shell thickness, $\alpha^{l}, \alpha^{2}=$ const Cartesian point coordinates of the domain $\Omega$. 


\subsection{Problem A}

Find a solution to the system (1) satisfying the boundary conditions (2)-(4).

We will study the boundary value problem $\mathrm{A}$ in a generalized setting. We consider the following conditions to be satisfied: a) $\Omega$ is a simply connected domain with the boundary $\Gamma \in C_{\beta}{ }^{l}$; b) external forces $R^{j}\left(j=\overline{1,3)}, L^{k}(k=1,2) \in L_{p}(\Omega)\right.$, the components of external forces $N^{I}, P^{I} \in C_{\beta}(\Gamma)$; here and further everywhere: $p>2,0<\beta<1$.

\subsection{Definition}

A generalized solution of problem A is the vector of generalized displacements $a=\left(w_{1}, w_{2}\right.$, $\left.w_{3}, \Psi_{1}, \Psi_{2}\right) \in W_{p}^{(2)}(\Omega), p>2$, which satisfies the system (1) and point wise boundary conditions (2)-(4) almost everywhere.

Let's consider a system of the first two equations in (1), in which the deflection $w_{3}$ is temporarily assumed to be fixed. The general solution of the system (1) has the form [18]:

$$
\omega_{0}(z)=w_{2}+i w_{1}=\Phi_{2}(z)+i T d\left[\Phi_{1}+T f\right](z), z=\alpha^{1}+i \alpha^{2}, f=\left(f_{1}+i f_{2}\right) / 2,
$$

where $\Phi_{l}(z) \in C_{\alpha}\left(\overline{\Omega)}, \Phi_{2}(z) \in C_{\alpha}{ }^{l}(\overline{\Omega)}\right.$ - arbitrary holomorphic functions;

$$
T f=-\frac{1}{\pi} \iint_{\Omega} \frac{f(\zeta)}{\zeta-z} d \xi d \eta, \zeta=\xi+i \eta, d[g]=d_{1} g+d_{2} \bar{g}, d_{j}=\left(\mu_{1}+(-1)^{j}\right) /\left(4 \mu_{1}\right), j=1,2 .
$$

We find the function $\Phi_{2}(z)$ from the boundary condition $w_{2}=0$ on $\Gamma$. We obtain the Riemann-Hilbert problem with the boundary condition for a holomorphic function $\Phi_{2}(z)$ in the domain $\Omega$ :

$$
\begin{gathered}
\operatorname{Re}\left[\Phi_{2}(t)\right]=-\operatorname{Re} i T d[\omega](t), t \in \Gamma, \\
\omega(z)=\Phi_{1}+T f(z)=w_{1 \alpha^{1}}+w_{2 \alpha^{2}}+i \mu_{1}\left(w_{2 \alpha^{1}}-w_{1 \alpha^{2}}\right) .
\end{gathered}
$$

Let us denote by the function $z=\varphi(\zeta)$ the conformal mapping of the single circle $\bar{K}:|\zeta|$

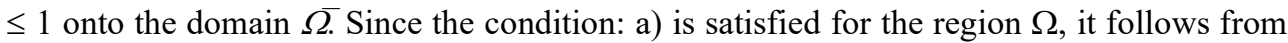
$\left[26\right.$, p. 25] that the function $\varphi(\zeta)$ belongs to the space $C_{\beta}{ }^{l}(\bar{K})$. In the boundary condition (7), we make the change $t \rightarrow \varphi(t), \Phi_{2}(\varphi(t)) \rightarrow \Phi_{2}(t)$, leaving the same designations for the new variables. Thus, in the single circle $K$ we arrive at the Riemann - Hilbert problem for a holomorphic function $\Phi_{2}(z)$ with the boundary condition:

$$
\operatorname{Re}\left[\Phi_{2}(t)\right]=-\operatorname{Re} i T d[\omega](\varphi(t)), t \in \partial K:|t|=1,
$$

where $d[g], T f$ are defined in (6). The solution of the problem (8) is given by the formula [27, p. 253]:

$$
\Phi_{2}(z)=\frac{1}{2 \pi} \int_{\partial K} \operatorname{Re} T d\left[\Phi_{1}+T f\right](\varphi(t)) \frac{t+z}{t-z} \frac{d t}{t}+i c_{0}, \quad z \in \bar{K},
$$

where $c_{0}$ an arbitrary real constant.

We find the holomorphic function $\Phi_{1}(z)$ using the boundary condition (3). The expressions of the functions $w_{1}, w_{2}$ from (6) are introduced into (3). Taking into account the ratios:

$$
\begin{gathered}
t^{\prime}=d t / d s=d\left(\alpha^{1}+i \alpha^{2}\right) / d s=-\alpha^{2}+i \alpha^{1}=i\left(\alpha^{1}+i \alpha^{2}\right)=i t, \\
d \alpha^{1} / d s=\operatorname{Re} t^{\prime}=\left(t^{\prime}+\overline{t^{\prime}}\right) / 2, \quad t \in \Gamma,
\end{gathered}
$$

the boundary condition (3) can be represented as:

$$
\operatorname{Re}\left\{t^{\prime} \Phi(t)\right\}=h_{2}(t), t^{\prime}=d t / d s, \quad t \in \Gamma,
$$

where

$$
\begin{aligned}
& h_{2}(t)=l\left(w_{3}\right)(t)+\operatorname{Re}\left\{t^{\prime} S d\left[\Phi_{1}\right]^{+}(t)\right\}-\operatorname{Re}\left\{\mu_{3} \bar{t}^{\prime} \Phi_{1}(t)\right\} / 2, \quad \mu_{3}=(1+\mu) /(2(1-\mu)), \\
& \left.l(t)=\varphi_{1}\left(w_{3}\right)(t) /(\mu-1)+\operatorname{Re}\left\{t^{\prime} S d[T f]^{+}(t)\right\}-\mu_{3} d \alpha^{1} / d s \operatorname{Re} T f(t)\right\} \equiv l\left[f\left(w_{3}\right) ; \varphi_{1}\left(w_{3}\right)\right] ;
\end{aligned}
$$


$\varphi_{I}(t)$ defined in (5). Through $S d\left[\Phi_{1}\right]^{+}(t)$ they denote the limit of the function $S d\left[\Phi_{1}\right](z)$ at $z \rightarrow t \in \Gamma$ from inside the region $\Omega$. $\Phi(t)$ the boundary value of the holomorphic function $\Omega$ :

$$
\Phi(z)=i \Phi_{2}{ }^{\prime}(z)+\mu_{3} \Phi_{1}(z) / 2 .
$$

Thus, for the function $\Phi(z)$ in an arbitrary domain $\Omega$ we have the Riemann-Hilbert problem with the boundary condition (11). We will reduce this problem to the problem in a single circle. Using the conformal mapping of the domain $\bar{\Omega}$ onto the single circle $\bar{K}$, we obtain:

$$
\begin{gathered}
t^{\prime}=\frac{d t}{d s}=\frac{d(\varphi(\tau))}{d s}=\frac{d \varphi(\tau)}{d \tau} \frac{d \tau}{d s}=\varphi^{\prime}(\tau) \tau^{\prime} \frac{1}{\left|\varphi^{\prime}(\tau)\right|}, \\
t=\varphi(\tau), \quad \tau=\tau(\sigma), \quad \tau \in \partial K:|\tau|=1, \quad \tau^{\prime}=d \tau / d \sigma .
\end{gathered}
$$

Therefore, leaving the previous designations for the new variables, taking into account (10) and (14), we arrive at the Riemann-Hilbert problem for a holomorphic function $\Phi(z) \varphi^{\prime}(z)$ in the single circle $\bar{K}$ with the boundary condition:

$$
\operatorname{Re}\left[t^{\prime} \varphi^{\prime}(t) \Phi(t)\right]=h_{2}(\varphi(t))\left|\varphi^{\prime}(t)\right|, \quad t \in \partial K:|t|=1 .
$$

Let us study the problem (15). The index of the problem (15) is -1 . Then, following [27, p. 253], the solution of the problem (15) has the following form:

$$
\Phi(z)=-\frac{1}{\pi \varphi^{\prime}(z)} \int_{\partial K} \frac{h_{2}(\varphi(t))\left|\varphi^{\prime}(t)\right|}{t-z} \frac{d t}{t}, \quad z \in \bar{K},
$$

in this case, they satisfy the condition of this problem solvability:

$$
\int_{\partial K} \frac{h_{2}(\varphi(t))\left|\varphi^{\prime}(t)\right|}{t} d t=0 .
$$

For $\Phi_{1}(z)$ from relation (13) we have:

$$
\Phi_{1}(z)=\left(\Phi(z)-i \Phi_{2}{ }^{\prime}(z)\right) 2 / \mu_{3}, \mathrm{z} \in \Omega .
$$

Let's transform the representation (18). To do this, you need to find $S d\left[\Phi_{1}\right]^{+}(t), \Phi_{2}{ }_{2}(z)$, $\Phi_{1}(z)$. The representation for $S d\left[\Phi_{1}\right]^{+}(t)$ is found in [22]. For the function $\Phi_{2}^{\prime}(z)(z \in \bar{K})$ from (9), using the representation $d[g]$ from (6) and taking into account that $((\mathrm{t}+\mathrm{z}) /(\mathrm{t}-\mathrm{z}))=2 \mathrm{t} /(\mathrm{t}-\mathrm{z})^{2}$ we have the following:

$$
\begin{gathered}
\Phi_{2}^{\prime}(z)=-\frac{d_{1}}{2 \pi \varphi^{\prime}(z)}\left[\int_{\partial K} \frac{T \Phi_{1}(\varphi(t))}{(t-z)^{2}} d t+\int_{\partial K} \frac{\overline{T \Phi_{1}(\varphi(t))}}{(t-z)^{2}} d t\right]- \\
-\frac{d_{2}}{2 \pi \varphi^{\prime}(z)}\left[\int_{\partial K} \frac{T \overline{\Phi_{1}(\varphi(t))}}{(t-z)^{2}} d t+\int_{\partial K} \frac{\bar{T} \Phi_{1}(\varphi(t))}{(t-z)^{2}} d t\right]-\frac{1}{\pi \varphi^{\prime}(z)} \int_{\partial K} \operatorname{Re} T d[T f](\varphi(t)) \frac{d t}{(t-z)^{2}} .
\end{gathered}
$$

Let us calculate all the integrals on the right-hand side of the representation (19). Taking into account the operator $T$ representation (6) and changing the variable $t \rightarrow \varphi(t)$, we obtain:

$$
T \Phi_{1}(\varphi(t))=-\frac{1}{\pi} \iint \frac{\Phi_{1}(\zeta)}{\zeta-\varphi(t)} d \xi d \eta=-\frac{1}{\pi} \iint_{\Omega} \frac{\Phi_{1}(\zeta)\left|\varphi^{\prime}(\zeta)\right|^{2}}{\varphi(\zeta)-\varphi(t)} d \xi d \eta, \quad t \in \partial K .
$$

Further, taking into account the representation for the function $\varphi_{0}(\tau ; z)=(\varphi(\tau)-\varphi(z)) /(\tau-z)$ and the Cauchy formula, we find the following integrals easily:

$$
\begin{gathered}
\int_{\partial K} \frac{1 / \varphi_{0}(\tau ; t)}{(t-\tau)(t-z)^{2}} d t=\frac{\pi i}{(z-\tau)^{2} \varphi^{\prime}(\tau)}-\frac{2 \pi i \varphi^{\prime}(z)}{[\varphi(z)-\varphi(\tau)]^{2}}, \\
\int_{\partial K} \frac{1 / \varphi_{0}(\zeta ; t)}{(t-\zeta)(1-\bar{z} t)^{2}} d t=\frac{2 \pi i}{\varphi^{\prime}(\zeta)(1-\bar{z} \zeta)^{2}}, \\
\int_{\partial K} \frac{1 / \varphi_{0}(\zeta ; t)}{(t-\zeta)(t-z)^{2}} d t=\frac{2 \pi i}{(z-\zeta)^{2} \varphi^{\prime}(\zeta)}-\frac{2 \pi i \varphi^{\prime}(z)}{[\varphi(z)-\varphi(\zeta)]^{2}}, \\
\int_{\partial K} \frac{1 / \varphi_{0}(\tau ; t)}{(t-\tau)(1-\bar{z} t)^{2}} d t=\frac{\pi i}{\varphi^{\prime}(\tau)(1-\tau \bar{z})^{2}} .
\end{gathered}
$$


Then the first integral in the formula (19), taking into account (20) and the representation Tf from (6), is transformed to the form:

$$
\begin{gathered}
\int_{\partial K} \frac{T \Phi_{1}(\varphi(t))}{(t-z)^{2}} d t=-\frac{1}{\pi} \int_{\partial K}\left(\iint_{K} \frac{\Phi_{1}(\zeta)\left|\varphi^{\prime}(\zeta)\right|^{2}}{\varphi(\zeta)-\varphi(t)} d \xi d \eta\right) \frac{d t}{(t-z)^{2}}= \\
=-\frac{1}{\pi} \int_{\partial K}\left(\iint_{K} \frac{\partial}{\partial \bar{\zeta}}\left(\frac{\Phi_{1}(\zeta) \varphi^{\prime}(\zeta) \overline{\varphi(\zeta)}}{\varphi_{0}(\zeta ; t)}\right) \frac{d \xi d \eta}{\zeta-t}\right) \frac{d t}{(t-z)^{2}} .
\end{gathered}
$$

Applying the formula (4.9) [26, p. 29], we have:

$$
\begin{gathered}
\int_{\partial K} \frac{T \Phi_{1}(\varphi(t))}{(t-z)^{2}} d t=\int_{\partial K}\left(\frac{\Phi_{1}(t) \overline{\varphi(t)}}{2}-\frac{1}{2 \pi i} \int_{\partial K} \frac{\Phi_{1}(\tau) \varphi^{\prime}(\tau) \overline{\varphi(\tau)}}{\varphi_{0}(\tau ; t)} \frac{d \tau}{\tau-t}\right) \frac{d t}{(t-z)^{2}}= \\
=\frac{1}{2} \int_{\partial K} \frac{\Phi_{1}(t) \overline{\varphi(t)}}{(t-z)^{2}} d t+\frac{1}{2 \pi i} \int_{\partial K}\left(\int_{\partial K} \frac{1 / \varphi_{0}(\tau ; t)}{(t-\tau)(t-z)^{2}} d t\right) \Phi_{1}(\tau) \varphi^{\prime}(\tau) \overline{\varphi(\tau)} d \tau .
\end{gathered}
$$

Then, taking into account (21), we obtain:

$$
\int_{\partial K} \frac{T \Phi_{1}(\varphi(t))}{(t-z)^{2}} d t=\int_{\partial K} \frac{\Phi_{1}(\tau) \overline{\varphi(\tau)}}{(\tau-z)^{2}} d \tau-\varphi^{\prime}(z) \int_{\partial K} \frac{\Phi_{1}(\tau) \varphi^{\prime}(\tau) \overline{\varphi(\tau)}}{\left[\varphi_{0}(z ; \tau)\right]^{2}(z-\tau)^{2}} d \tau .
$$

Let us transform the second integral in representation (19). First, for this we use (20), then we apply the formula (4.9) to the integral over the domain $T$ [10, p. 29], and then - the last formula in (21), and calculate the integral:

$$
\begin{gathered}
\int_{\partial K} \frac{T \Phi_{1}(\varphi(t))}{(\bar{t}-\bar{z})^{2}} d \bar{t}=\int_{\partial K}\left(\frac{1}{\pi} \iint_{K} \frac{\partial}{\partial \bar{\zeta}}\left(\frac{\Phi_{1}(\zeta) \varphi^{\prime}(\zeta) \overline{\varphi(\zeta)}}{\varphi_{0}(\zeta ; t)}\right) \frac{d \xi d \eta}{\zeta-t}\right) \frac{d t}{t^{2}(\bar{t}-\bar{z})^{2}}= \\
=\int_{\partial K}\left(\frac{1}{2 \pi i} \int_{\partial K} \frac{\Phi_{1}(\tau) \varphi^{\prime}(\tau) \overline{\varphi(\tau)}}{\varphi_{0}(\tau ; t)} \frac{d \tau}{\tau-t}-\frac{1}{2} \Phi_{1}(t) \overline{\varphi(t)}\right) \frac{d t}{t^{2}(\bar{t}-\bar{z})^{2}}= \\
=\frac{-1}{2 \pi i} \int_{\partial K}\left(\int_{\partial K} \frac{1 / \varphi_{0}(\tau ; t)}{(t-\tau)(1-\bar{z} t)^{2}} d t\right) \Phi_{1}(\tau) \varphi^{\prime}(\tau) \overline{\varphi(\tau)} d \tau-\frac{1}{2} \int_{\partial K} \frac{\Phi_{1}(t) \overline{\varphi(t)}}{(1-\bar{z} t)^{2}} d t=-\int_{\partial K} \frac{\Phi_{1}(t) \overline{\varphi(t)}}{(1-\bar{z} t)^{2}} d t .
\end{gathered}
$$

It's obvious that:

$$
\int_{\partial K} \frac{\overline{T \Phi_{1}(\varphi(t))}}{(t-z)^{2}} d t=\left(\overline{\int_{\partial K} \frac{T \Phi_{1}(\varphi(t))}{(\bar{t}-\bar{z})^{2}} d \bar{t}}\right)=-\int_{\partial K} \frac{\overline{\Phi_{1}(t)} \varphi(t)}{(1-\bar{t})^{2}} d \bar{t}=\int_{\partial K} \frac{\overline{\Phi_{1}(t)} \varphi(t)}{(t-z)^{2}} d t .
$$

Let us calculate the third integral in representation (19). Applying the formulas (20) and (21), we obtain:

$$
\begin{gathered}
\int_{\partial K} \frac{T \overline{\Phi_{1}(\varphi(t))}}{(t-z)^{2}} d t=-\frac{1}{\pi} \int_{\partial K}\left(\iint_{K} \frac{\overline{\Phi_{1}(\zeta)}\left|\varphi^{\prime}(\zeta)\right|^{2}}{\varphi(\zeta)-\varphi(t)} d \xi d \mu\right) \frac{d t}{(t-z)^{2}}= \\
=\frac{1}{\pi} \iint_{K}\left(\int_{\partial K} \frac{1 / \varphi_{0}(\zeta ; t)}{(t-\zeta)(t-z)^{2}} d t\right) \overline{\Phi_{1}(\zeta)}\left|\varphi^{\prime}(\zeta)\right|^{2} d \xi d \mu= \\
=2 i \iint_{K} \frac{\overline{\Phi_{1}(\zeta)}\left|\varphi^{\prime}(\zeta)\right|^{2}}{(z-\zeta)^{2} \varphi^{\prime}(\zeta)} d \xi d \mu-2 i \varphi^{\prime}(z) \iint_{K} \frac{\overline{\Phi_{1}(\zeta)}\left|\varphi^{\prime}(\zeta)\right|^{2}}{[\varphi(z)-\varphi(\zeta)]^{2}} d \xi d \mu .
\end{gathered}
$$

Let $K_{\varepsilon}$ the area obtained from the area $K$ by throwing out the circle centered at the point $z:|\zeta-z| \leq \varepsilon$ with the boundary $\partial K \varepsilon=\partial K \cup \gamma_{\bar{\varepsilon}}$. Then we have:

$$
\iint_{K} \frac{\overline{\Phi_{1}(\zeta)}\left|\varphi^{\prime}(\zeta)\right|^{2}}{(z-\zeta)^{2} \varphi^{\prime}(\zeta)} d \xi d \mu=\iint_{K} \frac{\overline{\Phi_{1}(\zeta) \varphi^{\prime}(\zeta)}}{(z-\zeta)^{2}} d \xi d \mu=-\lim _{\varepsilon \rightarrow 0} \iint_{K_{\varepsilon}} \frac{\partial}{\partial \zeta}\left(\overline{\frac{\Phi_{1}(\zeta) \varphi^{\prime}(\zeta)}{\zeta-z}}\right) d \xi d \mu .
$$

Using the formula (4.7) [26, p. 28], we calculate the following integral:

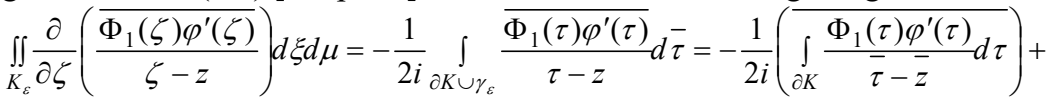

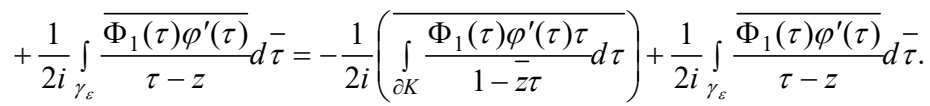


Here the first term is equal to zero, since the function $\Phi_{1}(\tau) \varphi^{\prime}(\tau) \tau /(1-\overline{z \tau})$ is the boundary value of the function $\Phi_{1}(\zeta) \varphi^{\prime}(\zeta) \zeta /(1-\bar{z} \zeta)$, holomorphic by $\zeta$ in $\bar{K}$. We use the replacement for the second term:

$$
\frac{1}{2 i} \int_{\gamma_{\varepsilon}} \overline{\frac{\Phi_{1}(\tau) \varphi^{\prime}(\tau)}{\tau-z}} d \bar{\tau}=\left[\begin{array}{c}
\tau=z+\varepsilon e^{i \theta} \\
\bar{\tau}=\bar{z}+\varepsilon e^{-i \theta} \\
d \bar{\tau}=-i \varepsilon e^{-i \theta} d \theta
\end{array}\right]=-\frac{1}{2 i} \int_{0}^{2 \pi} \frac{\overline{\Phi_{1}\left(z+\varepsilon e^{i \theta}\right) \varphi^{\prime}\left(z+\varepsilon e^{i \theta}\right)}}{e^{2 i \theta}} d \theta
$$

and passing to the limit at $\varepsilon \rightarrow 0$, we obtain

$$
\lim _{\varepsilon \rightarrow 0} \frac{1}{2 i} \int_{\gamma_{\varepsilon}} \frac{\overline{\Phi_{1}(\tau) \varphi^{\prime}(\tau)}}{\tau-z} d \bar{\tau}=-\frac{1}{2 i} \overline{\Phi_{1}(z) \varphi^{\prime}(z)} \int_{0}^{2 \pi} e^{-2 i \theta} d \theta=0 .
$$

Therefore, the integral over the region $K$ :

$$
\iint_{K} \frac{\overline{\Phi_{1}(\zeta)}\left|\varphi^{\prime}(\zeta)\right|^{2}}{(z-\zeta)^{2} \varphi^{\prime}(\zeta)} d \xi d \mu=0
$$

Similarly, using the formula (4.7) [26, p. 28] in the case of the domain $K_{\varepsilon}$, we find the integral:

$$
\begin{gathered}
\iint_{K} \frac{\overline{\Phi_{1}(\zeta)}\left|\varphi^{\prime}(\zeta)\right|^{2}}{[\varphi(z)-\varphi(\zeta)]^{2}} d \xi d \mu=-\lim _{\varepsilon \rightarrow 0} \iint_{K_{\varepsilon}} \frac{\partial}{\partial \zeta}\left(\frac{\overline{\Phi_{1}(\zeta) \varphi^{\prime}(\zeta)} / \varphi_{0}(\zeta ; z)}{\zeta-z}\right) d \xi d \mu= \\
=\frac{1}{2 i} \int_{\partial K} \frac{\overline{\Phi_{1}(\tau) \varphi^{\prime}(\tau)}}{\varphi_{0}(\tau ; z)(\tau-z)} d \bar{\tau}-\frac{1}{2 i} \lim _{\varepsilon \rightarrow 0} \int_{\gamma_{\varepsilon}} \frac{\overline{\Phi_{1}(\tau) \varphi^{\prime}(\tau)}}{\varphi_{0}(\tau ; z)(\tau-z)} d \bar{\tau}=-\frac{1}{2 i} \int_{\partial K} \frac{\bar{\Phi}_{1}(\tau) \varphi^{\prime}(\tau) \tau}{\varphi_{0}(\tau ; z)(\tau-z)} d \tau,
\end{gathered}
$$

because $\lim _{\varepsilon \rightarrow 0} \int_{\gamma_{\varepsilon}} \frac{\overline{\Phi_{1}(\tau) \varphi^{\prime}(\tau)}}{\varphi_{0}(\tau ; z)(\tau-z)} d \bar{\tau}=0$.

Thus, for the third integral in representation (19), we have:

$$
\int_{\partial K} \frac{T \overline{\Phi_{1}(\varphi(t))}}{(t-z)^{2}} d t=\varphi^{\prime}(z) \int_{\partial K} \frac{\overline{\Phi_{1}(\tau) \varphi^{\prime}(\tau) \tau}-{ }^{2}}{\varphi_{0}(\tau ; z)(\tau-z)} d \tau .
$$

Now we calculate the fourth integral in the representation (19). Taking into account (20), (21), the formula (4.7) [26, p. 28] and Cauchy's formula, we obtain:

$$
\begin{gathered}
\int_{\partial K} \frac{\bar{T} \Phi_{1}(\varphi(t))}{(t-z)^{2}} d t=\int_{\partial K}\left(-\frac{1}{\pi} \iint_{K} \frac{\Phi_{1}(\zeta)\left|\varphi^{\prime}(\zeta)\right|^{2}}{\overline{\varphi(\zeta)}-\overline{\varphi(t)}} d \xi d \mu\right) \frac{d t}{(t-z)^{2}}= \\
=-\frac{1}{\pi} \iint_{K}\left(\int_{\partial K} \frac{\overline{1 / \varphi_{0}(t ; \zeta) d t}}{(t-\zeta)(1-\bar{z} t)^{2}}\right) \Phi_{1}(\zeta)\left|\varphi^{\prime}(\zeta)\right|^{2} d \xi d \mu=2 i \iint_{K} \frac{\Phi_{1}(\zeta) \varphi^{\prime}(\zeta)}{(1-z \bar{\zeta})^{2}} d \xi d \mu= \\
=\frac{2 i}{z} \iint_{K} \frac{\partial}{\partial \bar{\zeta}}\left(\frac{\Phi_{1}(\zeta) \varphi^{\prime}(\zeta)}{1-z \bar{\zeta}}\right) d \xi d \mu=\frac{1}{z} \int_{\partial K} \frac{\tau \Phi_{1}(\tau) \varphi^{\prime}(\tau)}{\tau-z} d \tau=2 \pi i \Phi_{1}(z) \varphi^{\prime}(z) .
\end{gathered}
$$

Substituting the found expressions of the integrals into the formula (19), we obtain the following representation for $\Phi_{2}{ }^{\prime}(z)$ :

$$
\begin{gathered}
\Phi_{2}^{\prime}(z)=\frac{d_{1}}{2 \pi} \int_{\partial K} \frac{\Phi_{1}(\tau) \phi^{\prime}(\tau) \overline{\phi(\tau)}}{\left[\phi_{0}(z ; \tau)\right]^{2}(z-\tau)^{2}} d \tau-\frac{d_{1}}{2 \pi \phi^{\prime}(z)} \int_{\partial K} \frac{\Phi_{1}(\tau) \overline{\phi(\tau)}}{(\tau-z)^{2}} d \tau- \\
-\frac{d_{1}}{2 \pi \varphi^{\prime}(z)} \int_{\partial K} \frac{\overline{\Phi_{1}(\tau) \varphi(\tau)}}{(\tau-z)^{2}} d \tau-\frac{d_{2}}{2 \pi} \int_{\partial K} \frac{\left.\overline{\Phi_{1}(\tau) \varphi^{\prime}(\tau) \tau}\right)^{2}}{\varphi_{0}(\tau ; z)(\tau-z)} d \tau-i d_{2} \Phi_{1}(z)- \\
-\frac{1}{\pi \varphi^{\prime}(z)} \int_{\partial K} \frac{\operatorname{Re} T d[T f](\varphi(\tau)) d \tau}{(\tau-z)^{2}}, \quad z \in \bar{K} .
\end{gathered}
$$


To find $\Phi(z)$ we substitute in the formula (12) according to formulas (14) and put the resulting expression into the formula (16). After cumbersome calculations for the function $\Phi(z)$ we obtain the following representation:

$$
\begin{gathered}
\Phi(z)=\frac{i d_{1}}{2 \pi} \int_{\partial K} \frac{\Phi_{1}(\tau) \phi^{\prime}(\tau) \overline{\phi(\tau)}}{\left[\phi_{0}(z ; \tau)\right]^{2}(z-\tau)^{2}} d \tau-\frac{i d_{1}}{2 \pi \phi^{\prime}(z)} \int_{\partial K} \frac{\Phi_{1}(\tau) \overline{\phi(\tau)}}{(\tau-z)^{2}} d \tau- \\
-\frac{i d_{2}}{2 \pi} \int_{\partial K} \frac{\overline{\Phi_{1}(\tau) \varphi^{\prime}(\tau) \tau}-2}{\varphi_{0}(\tau ; z)(\tau-z)} d \tau-\frac{i d_{1}}{2 \pi \varphi^{\prime}(z)} \int_{\partial K} \frac{\overline{\Phi_{1}(\tau)} \varphi(\tau)}{(\tau-z)^{2}} d \tau-\frac{1}{\pi \varphi^{\prime}(z)} \int_{\partial K} \frac{l\left(w_{3}\right)(\varphi(\tau))\left|\varphi^{\prime}(\tau)\right| d \tau}{\tau(\tau-z)} .
\end{gathered}
$$

We substitute the expressions $\Phi_{2}{ }^{\prime}(z)$ from (22) and $\Phi(z)$ from (23) into the formula (18). After cumbersome transformations, we get the following representation for $\Phi_{1}(z)$ :

$$
\begin{gathered}
\Phi_{1}(z) \equiv \Phi_{1}\left[l\left(w_{3}\right)\right](z)=2(\mu-1) S_{\partial K}(\operatorname{Re} T d[T f](\varphi(\tau)))(z)+ \\
+\frac{\mu-1}{\pi} \int_{\partial K} \frac{l\left(w_{3}\right) \varphi(t)\left|\varphi^{\prime}(t)\right|}{t(t-z)} d t, \quad z \in K, S_{\partial K} f(z)=\frac{1}{2 \pi i} \int_{\partial K} \frac{f(t)}{(t-z)^{2}} d t,
\end{gathered}
$$

the operator $l\left(w_{3}\right)$ is introduced in the formula (12).

Let us denote via $\zeta=\Psi(z)$ the function inverse to the function $z=\varphi(\zeta)$. Known [26, p. 25], that $\Psi(z) \in C_{\beta}{ }^{l}(\Omega)$. Then the $\Phi_{1}(\Psi(z)) \in W_{p}^{(l)}(\Omega), 2<p<2 /(1-\beta)$.

Substituting the expression of the function $\Phi_{1}(z)$ from the formula (24) into the formula (9), we obtain the representations for $\Phi_{2}(\Psi(z))$ of the following form:

$$
\begin{gathered}
\Phi_{2}(\psi(z))=\Phi_{2}\left[l\left(w_{3}\right)\right](\psi(z))+i c_{0}, \quad z \in \Omega, \\
\Phi_{2}\left[l\left(w_{3}\right)\right](\psi(z))=-\frac{1}{2 \pi} \int_{\partial K}\left(\operatorname{Re} T d\left[\Phi_{1}\left[l\left(w_{3}\right)\right]\right](t)+\operatorname{Re} T d[T f](t)\right) \frac{t+\psi(z)}{t-\psi(z)} \frac{d t}{t} .
\end{gathered}
$$

Substituting the representations (24), (25) into the formula (6), for the functions $w_{1}, w_{2}$, satisfying the system of the first two equations in (1) and the boundary conditions in (2), (3), under condition (17) we obtain the desired representation:

$$
\begin{gathered}
\omega_{0}(z)=H_{0} w_{3}(z)+i c_{0}, \quad z \in \Omega, \\
H_{0} w_{3}(z)=\Phi_{2}\left[l\left(w_{3}\right)\right](\psi(z))+i \operatorname{Td}\left[\Phi_{1}\left[l\left(w_{3}\right)\right](\psi(\zeta))+T f\left(w_{3}\right)(\zeta)\right](z) .
\end{gathered}
$$

To transform the solvability condition (17), the function $h_{2}(\varphi(t))$ is replaced by the formula (12). Further, applying the formula (14) and Cauchy's formula, we can easily reduce the condition (17) to the form $\int_{\partial K}\left(l\left(w_{3}\right)(\varphi(t))\left|\varphi^{\prime}(t)\right| / t\right) d t=0$, which, in turn, is transformed to the following final form:

$$
\int_{\Gamma} P^{1}(s) d s+\iint_{\Omega} R^{1} d \alpha^{1} d \alpha^{2}=0
$$

where $P^{l}(s), R^{l}\left(\alpha^{l}, \alpha^{2}\right)$ the components of the external load.

Further, from the last two equations of the system (1), we find the functions $\Psi_{1}, \Psi_{2}$, that satisfy the condition $\Psi_{2}=0$ and the term (4) on the boundary $\Gamma$. Note that the structure of the left-hand sides of the last two equations of the system (1) and boundary condition (4) is the same as for the functions $w_{1}, w_{2}$; they differ only in the right-hand sides. Therefore, for the functions $\Psi_{1}, \Psi_{2}$ with fixed right-hand sides, we find similar representations:

$$
\begin{aligned}
& \psi=\psi_{2}+i \psi_{1}=H_{0}\left[g+\widetilde{\psi} ; l\left[g+\widetilde{\psi} ; \widetilde{\varphi}_{1}\right]\right]+i c_{1}, \\
& g \equiv g\left(w_{3}\right)=\left(g_{1}+i g_{2}\right) / 2, \widetilde{\psi}=k_{0}\left(\psi_{2}+i \psi_{1}\right) / 2,
\end{aligned}
$$

where the functions $g_{j} \equiv g_{j}\left(w_{3}\right)$ are defined in (5), the operator $H_{0}[f ; g]-$ in (26), $c_{l}$ an arbitrary real constant.

Besides, the solvability condition must be satisfied:

$$
\beta_{1}\left(\int_{\Gamma} N^{1}(s) d s+\iint_{\Omega} L^{1} d \alpha^{1} d \alpha^{2}\right)-k_{0} \iint_{\Omega} \psi_{1} d \alpha^{1} d \alpha^{2}=0,
$$

where $N^{1}, L^{1}$ the components of the external load.

Taking into account that the operator $H_{0}\left[g+\widetilde{\psi} ; l\left[g+\widetilde{\psi} ; \widetilde{\varphi}_{1}\right]\right]=H_{0}\left[g ; \widetilde{\varphi}_{1}\right]+H_{0}[\tilde{\psi} ; 0]$, of the function $\Psi_{1}, \Psi_{2}$ from (28) it can be represented in the form of an operator equation 
$\psi-K_{0} \psi=H_{0}\left[g ; \widetilde{\varphi}_{1}\right]+i c_{1}, K_{0} \psi=H_{0}[\widetilde{\psi} ; 0]$, Further, proceeding as in [22], we reduce this equation to the following form:

$$
\psi \equiv \psi\left(w_{3}\right)=\left(I-K_{0}\right)^{-1} H_{0}\left[g\left(w_{3}\right) ; \tilde{\varphi}_{1}\right]
$$

In this case, the solvability condition (29) will be satisfied identically. Thus, we obtain an unambiguous representation for the functions $\Psi_{1}, \Psi_{2}$ through the deflection $w_{3}$ of the form (30).

Problem A will be reduced to a single operator equation for the function $w_{3}$. For this, in the third equation of system (1), the functions $w_{1}, w_{2}, \Psi_{1}, \Psi_{2}$ and their first-order derivatives are replaced by expressions from (26), (30). Then we arrive at a nonlinear second-order partial differential equation with respect to $w_{3}$ of the form:

where

$$
w_{3 \alpha^{1} \alpha^{1}}+w_{3 \alpha^{2} \alpha^{2}}+K_{1} w_{3}+G_{1} w_{3}=0
$$

$$
\begin{gathered}
K_{1} w_{3}=\psi_{11 \alpha^{1}}\left(w_{3}\right)+\psi_{21 \alpha^{2}}\left(w_{3}\right)+\left\{k_{3} w_{11 \alpha^{1}}\left(w_{3}\right)+k_{4} w_{21 \alpha^{2}}\left(w_{3}\right)-k_{5} w_{3}\right\} /\left(k^{2} \mu_{1}\right), \\
G_{1} w_{3}=\psi_{10 \alpha^{1}}+\psi_{20 \alpha^{2}}+\left\{k_{3} w_{12 \alpha^{1}}\left(w_{3}\right)+k_{4} w_{22 \alpha^{2}}\left(w_{3}\right)+\left(k_{3} w_{3 \alpha^{1}}^{2}+k_{4} w_{3 \alpha^{2}}^{2}\right\} / 2+\right. \\
\left.+\beta_{2}\left[\left(T^{\lambda \mu} w_{3 \alpha^{\lambda}}\right)_{\alpha^{\mu}}+R^{3}\right]\right\} /\left(k^{2} \mu_{1}\right) .
\end{gathered}
$$

Thus, finding the solution to the problem A was reduced to the equation (31) solution for the function $w_{3}$ with the condition $w_{3}=0$ on the boundary $\Gamma$. The problem is the equivalent equation:

$$
w_{3}+G * w_{3}=0, G * w_{3}=(I+K)^{-1} G w_{3}, G w_{3}=\iint_{\Omega} H(\zeta, z) G_{1} w_{3}(\zeta) d \zeta d \eta
$$

$H(\zeta, z)=\frac{1}{2 \pi} \ln \frac{|\psi(z)-\psi(\zeta)|}{|1-\psi(z) \overline{\psi(\zeta)}|}$ - the harmonic Green's function for the domain $\Omega$, the operator $G_{1} w_{3}$ is defined in the formula (31). The operator $G_{*} w_{3}$ is nonlinear bounded operator in $W_{p}^{(2)}(\Omega)$. Moreover, for any two values of $w_{3}{ }^{j} \in W_{p}^{(2)}(\Omega), j=1.2$, belonging to the ball $\left\|w_{3}\right\|_{W_{p}^{(2)}(\Omega)}<r$, the following estimate is fair:

$$
\begin{gathered}
\left\|G_{*} w_{3}^{1}-G_{*} w_{3}^{2}\right\|_{W_{p}^{(2)}(\Omega)} \leq q_{*}\left\|w_{3}^{1}-w_{3}^{2}\right\|_{W_{p}^{(2)}(\Omega)} \\
q_{*}=c\left\|\left(I+K_{0}\right)^{-1}\right\|_{W_{p}^{(2)}(\Omega)}\left[q_{0}+(1+r) r\right], q_{0}=\sum_{\lambda, \mu=1}^{2}\left\|T^{\lambda \mu}(0)\right\|_{C(\bar{\Omega})}+\sum_{\lambda=1}^{2}\left\|R^{\lambda}\right\|_{L_{p}(\Omega)} .
\end{gathered}
$$

\section{Results and discussion}

Let us assume that the external forces acting on the shell and the radius $r$ of the sphere are such that the following conditions are satisfied:

$$
q^{*}<1,\left\|G_{*}(0)\right\|_{W_{p}^{(2)}(\Omega)}<\left(1-q_{*}\right) r .
$$

Then the equation (32) in the ball $\left\|w_{3}\right\|_{W_{p}^{(2)}(\Omega)}<r$ has a unique solution $w_{3} \in W_{p}^{(2)}(\Omega)$, $2<p<2 /(1-\beta)$ according to [28]. Substituting the found solution into the formulas (26), (30), we find the functions $w_{1}, w_{2}, \Psi_{1}, \Psi_{2} \in W_{p}^{(2)}(\Omega)$. Moreover, the functions $w_{2}, \Psi_{1}, \Psi_{2}$ are determined uniquely, and the function $w_{1}$ up to a constant term $c_{0}$. The condition (27) is not only sufficient, but also a necessary condition for the solvability of the problem A.

\section{Conclusion}

Let conditions a), b) from the problem A, and the inequality (33) be satisfied. Then, to solve the problem A, it is necessary and sufficient that the condition (27) be satisfied. In the case of its fulfillment, the problem has a generalized solution a $=\left(w_{1}, w_{2}, w_{3}, \Psi_{l}, \Psi_{2}\right) \in W_{p}^{(2)}(\Omega)$, 
$2<p<2 /(1-\beta)$, in which the components $w_{2}, w_{3}, \Psi_{1}, \Psi_{2}$ are determined uniquely, and the component $w_{1}$ up to a constant term.

\section{References}

1. R.B. Rikards. Finite element method in the theory of shells and plates, Riga (1988).

2. V.F. Kirichenko, J. Awrejcewicz, A.F. Kirichenko, A.V. Krysko, V.A. Krysko. International Journal of Non-Linear Mechanics 74 (2015). DOI: 10.1016/j.ijnonlinmec.2015.03.011.

3. S.S. Gavryushin, A.S. Nikolaeva. Mechanics of Solids 51 3, (2016). DOI: 10.3103/ S0025654416030110.

4. V.N. Paimushin. Mechanics of Composite Materials 53 5, (2017). DOI: 10.1007/s11 029017-9691-7.

5. R.A. Kayumov. Mechanics of Solids 52 5, (2017). DOI: 10.3103/S0025654417050120.

6. V.N. Paimushin, R.A. Kayumov, S.A. Kholmogorov, V.M. Shishkin. Russian Mathematics 62 6, (2018). DOI: 10.3103/S1066369X18060087.

7. V.N. Paimushin, S.A. Kholmogorov, R.A. Kayumov. Uchenye zapiski Kazanskogo universiteta Seriya fiziko-matematicheskie nauki 159 4, (2017).

8. R.A. Kayumov, B.F. Tazyukov, F.R. Shakirzyanov, I.Z. Mukhamedova. Lobachevskii Journal of Mathematics 40 3, (2019). DOI: 10.1134/S19 95080219030119.

9. R.A. Kayumov, B.F. Tazyukov, I.Z. Mukhamedova. $11^{\text {th }}$ International conference on «Mesh methods for boundary-value problems and applications». Kazan: Ins. of Phys. Publ., (2016). DOI: 10.1088/1757-899X/158/1/012051.

10. I.I. Vorovich, Mathematical problems of nonlinear theory of shallow shells. Moscow, (1989).

11. N.F. Morozov. Selected two-dimensional problems of elasticity theory. Leningrad, (1978).

12. I.B. Badriev, V.V. Banderov, G.Z. Garipova, M.V. Makarov, R.R. Shagidullin. Applied Mathematical Sciences 9 82, (2015). DOI: 10.12988/ams.2015.54358.

13. I.B. Badriev, M.V. Makarov, V.N. Paimushin. Russian Mathematics 59 10, (2015). DOI: 10.3103/S1066369X15100072.

14. M.M. Karchevskii. Uchenye zapiski Kazanskogo universiteta Seriya fiziko matematicheskie nauki 158 3, (2016). DOI: 10.1088/1757-899X/158/1/012048.

15. M.M. Karchevskii. $11^{\text {th }}$ International conference on «Mesh methods for boundary-value problems and applications». Kazan: Ins. of Phys. Publ., (2016).

16. I.B. Badriev, M.V. Makarov, V.N. Paimushin. Russian Mathematics 61 1, (2017). DOI: 10.3103/S1066369X1701008X.

17. V.N. Paimushin, S.A. Kholmogorov, I.B. Badriev. Lobachevskii Journal of Mathematics 40 3, (2019). DOI: 10.1134/S1995080219030156.

18. S.N. Timergaliev, A.N. Uglov, L.S. Kharasova. Russian Mathematics 59 5, (2015). DOI: 10.3103/S1066369X15050060.

19. M.G. Ahmadiev, S.N. Timergaliev, L.S. Kharasova. Journal of Siberian Federal University. Mathematics \& Physics 9 2, (2016).

20. S.N. Timergaliev, L.S. Kharasova. Differential Equations 52 5, (2016).

21. S.N. Timergaliev. Russian Mathematics 61 4, (2017). DOI: 10.3103/S1066369X17040089.

22. S.N. Timergaliev, L.S. Kharasova. IOP Conf. Series: Materials Science and Engineering 158, (2016).

23. S.N. Timergaliev. Differential Equations 55 2, (2019). DOI: 10.1134/S0012266119020095.

24. L.S. Kharasova. IOP Conf. Series: Journal of Physics 1158, (2019).

25. K.Z. Galimov. Principles of the nonlinear theory of thin shells, Kazan, (1975).

26. I.N. Vekua. Generalized analytic function, Moscow, (1988).

27. F.D. Gakhov, Boundary-value problems, Moscow, (1963).

28. M.A. Krasnosel'skii. Topological methods in the theory of nonlinear integral equations, Moscow, (1956). 